\title{
Dynamical Evolution of the Mass Function of the Globular Cluster System from Fokker-Planck Calculations: Preliminary Results
}

\author{
Jihye Shin and Sungsoo S. Kim \\ Department of Astronomy and Space Science, Kyung Hee University, Yongin-shi, \\ Kyungki-do 449-701, South Korea \\ email: jhshin@ap1.khu.ac.kr
}

\begin{abstract}
Using anisotropic Fokker-Planck models, we calculate the evolution of mass and luminosity functions of the Galactic globular cluster system. Our models include two-body relaxation, binary heating, tidal shocks, dynamical friction, and stellar evolution. We perform Fokker-Planck simulations for a large number of virtual globular clusters and synthesize these results to study the relation between the initial and present GCMFs.
\end{abstract}

Keywords. Globular cluster, Fokker-Planck

Most recently, Fall \& Zhang (2001; FZ hereafter) have semi-analytically calculated the evolution of the mass function of globular clusters (GCMF) in the Milky Way by considering the rate of mass loss by each process linearly and independently.
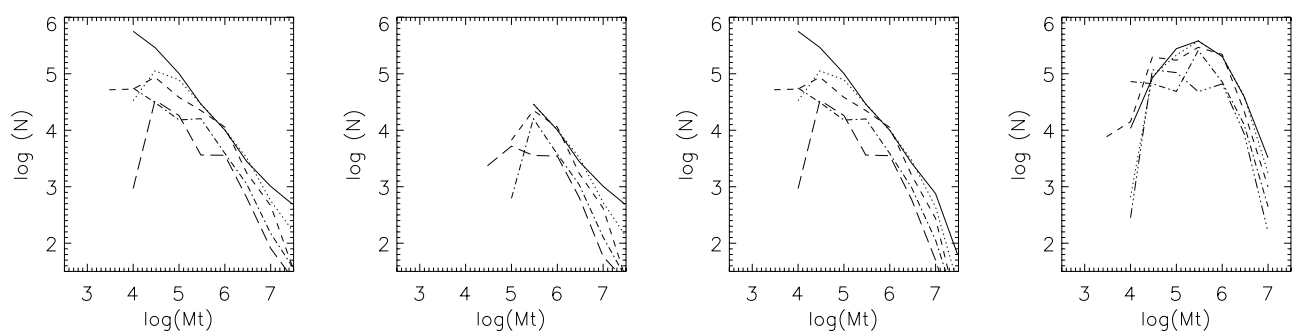

Figure 1. Evolution of the GCMF for four different initial cluster system mass function (a power law, truncated power law, a Schechter function, a lognormal function). Each mass function is plotted for $\mathrm{t}=0,1.5,3,6,12$ Gyr (Solid, dotted, dashed, dash-dot, long dashes).

Here, we adopted Fokker-Planck (F-P) codes developed by Takahashi \& Lee (2000) that would give us more accurate results than a semi-analytic method. We performed a large set of F-P calculations for various initial conditions that were similar to those in FZ. We considered the stellar evolution, two-body relaxation and dynamical friction. Our results qualitatively agree with FZ, but low-mass clusters evolve more quickly than $\mathrm{FZ}$, and the GCMF at $\mathrm{t}=12 \mathrm{Gyr}$ has a peak at smaller masses (around $3 \times 10^{4} \sim 10^{5} \mathrm{M} \odot$ ).

We will include the effects of bulge and disk shock in the near future.

\section{References}

Fall, S.M. \& Zhang, Q. 2001, ApJ 561, 751.

Takahashi, K. \& Lee, H.M. 2000, ApJ 316, 671. 